\title{
Evaluation of anterior chamber volume using Pentacam and anterior segment optical coherence tomography among normal subjects
}

\author{
Vijaya Anandan ${ }^{1,2}$, Rekha Srinivasan ${ }^{1}$, Rashima Asokan ${ }^{1,2}$, Ronnie \\ George ${ }^{2}$ \\ ${ }^{1}$ Elite School of Optometry, Chennai, India; ${ }^{2}$ Medical Research Foundation, \\ Chennai, India
}

\begin{abstract}
Aim: To compare the anterior chamber volume measurements obtained with Pentacam and derived from anterior segment optical coherence tomography.

Design: Cross-sectional study.

Methods: We included normal subjects who underwent a comprehensive eye examination including refraction, keratometry, Goldmann applanation tonometry, gonioscopy, anterior segment optical coherence tomography (AS-OCT) (Carl Zeiss Meditec Inc.; Dublin, CA, USA) and Pentacam (Oculus Inc.; Lynnwood, WA, USA). Fifty scans were selected for Pentacam and 12 images were selected for calculation of anterior chamber volume. Only the right eye was considered for analysis.

Results: One-hundred and nineteen eyes of 119 subjects were included for analysis. The mean age of the subjects was $42.58 \pm 13.15$ years, of which 74 were female and 45 were male. The mean anterior chamber volume measured using AS-OCT was $119.17 \pm 26.56$ $\mathrm{mm} 3$ and with Pentacam was $131.29 \pm 34.26 \mathrm{~mm} 3$. The comparison of means between the two modalities was statistically significant $(t=-8.857$, Mean Difference $(M D)=12.11$, $95 \% \mathrm{Cl}:(4.29,19.95), p=0.003)$. Bland-Altman plot showed poor agreement between the chamber volume measurements obtained by Pentacam and AS-OCT with MD of 12.1 $\mathrm{mm} 3(95 \% \mathrm{Cl}: 41.4$ to -17.1$)$ and intra-class correlation between the two instruments was 0.94 (95\% Cl: 0.91, 0.96) $(p<0.0001)$.

Conclusion: The anterior chamber volume can be measured using Pentacam as well as AS-OCT since these measurements were reliable. However, these measurements were not interchangeable due to poor levels of agreement.
\end{abstract}

Keywords: anterior chamber volume, Pentacam, optical coherence tomography (OCT)

\section{Introduction}

Evaluation of anterior chamber parameters is essential for ophthalmic evaluation. Slit lamp examination is of help for anterior segment examination. Objective and

Correspondence: Dr Ronnie George, Medical Research Foundation, Sankara Nethralaya, 18, College Road, Chennai 600 006, India.

E-mail: drrg@snmail.org 
quantitative information regarding the angle structures can be found with the help of gonioscopic lenses. However, the procedure is examiner dependent and the findings are subject to variability. Furthermore, they do not provide quantitative information regarding the angle structures and angle parameters for further follow-up examination. New anterior segment imaging techniques overcomes these difficulties. Anterior chamber measurements can be measured using biometry and ultrasound biomicroscopy as contact procedures and anterior segment optical coherence tomography (AS-OCT) and Pentacam as non-contact procedures. These instruments give us the quantitative measurements of cornea, anterior chamber, iris, angle structures, and lens. The information provided by these techniques helps clinicians in understanding the quantitative and qualitative information about the anatomic relationships of the anterior segment. These instruments provide information such as angle, depth measurement, and volume of the anterior segment.

The assessment of anterior chamber volume (ACV) helps us in understanding the mechanism of aqueous humour dynamics and has relevance in primary angle-closure glaucoma and pigmentary glaucoma. Fluorometry is considered to be the gold standard in assessing ACV. ${ }^{1}$ This procedure is invasive and actually gives aqueous volume rather than ACV. The available devices used for measuring ACV are the Pentacam (Oculus Inc.; Lynnwood, WA, USA) and the Visante OCT (Carl Zeiss Meditec Inc.; Dublin, CA, USA).

Pentacam uses the Scheimpflug principle to obtain images of the anterior segment. It takes up to 50 scans of the anterior segment in less than 2 seconds. It calculates data for corneal topography (anterior and posterior corneal surface) and thickness, anterior chamber depth, lens opacification, and lens thickness. The Pentacam-Scheimpflug requires minimal experience for image acquisition. When correct alignment and focus with the patient's cornea has been achieved, it has an inbuilt function which automatically gets started.

AS-OCT is a non-contact procedure that is performed in the sitting position. With a spatial resolution of $10-20 \mu \mathrm{m}$, the Visante OCT is a non-contact optical system that uses a $1310 \mathrm{~nm}$ super luminescent diode to image the anterior chamber of the eye. It is easy to operate and allows view of both angles simultaneously and the iris-lens interaction, providing anterior segment scans, high-resolution corneal and anterior chamber angle scans, and pachymetry maps both relative and differential at a rate of up to $2048 \mathrm{~A}$-scans per second. It gives information on depth, width, and anterior chamber angle. It has an optical axial resolution of up to $18 \mathrm{~mm}$ and transverse resolution of up to $60 \mathrm{~mm}$. Imaging of opaque corneas is possible and minimal experience is required for image acquisition. ACV can be derived from the measurements using custom-designed software.

Various studies have reported the ACV measurements with Pentacam and 
AS-OCT. ${ }^{2,3}$ The repeatability and reproducibility in measuring anterior chamber parameters between these studies remains unclear, with $\mathrm{Yi}$ et al. ${ }^{4}$ and Labiris et al. ${ }^{5}$ reporting good agreement with Pentacam and AS-OCT measurements, but Fu et al. ${ }^{7,10}$ reporting poor agreement between these devices. To the best of our knowledge, no studies have reported on the measurement of ACV using AS-OCT and Pentacam in the Indian population. Thus, the aim of our study is to understand the comparison of ACV measurements using Visante OCT and Pentacam.

\section{Materials and methods}

\section{Subject selection}

The study was a cross-sectional study. Normal subjects with no evidence of previous ocular trauma and ocular surgeries were included in the study. All the normal participants underwent a comprehensive eye examination including refraction, keratometry (KMS-6; Appaswamy Associates; Chennai, India), Goldmann applanation tonometry, gonioscopy, Visante AS-OCT, and Pentacam. Sample size calculation was based on a study by Fu et al. The study was approved by the Ethics Committee and adhered to the tenets of the Declaration of Helsinki and written informed consent was acquired from all the participants.

\section{Measurement of ACV using Pentacam}

ACV was assessed by Pentacam. Fifty scans were selected for the better assessment of the anterior chamber parameters. The test was done without dilation. From the recorded Scheimpflug images, the system calculates a Virtual Eye of the anterior eye segment. From this, all further information such as the anterior chamber analysis, topography, pachymetry, etc. are calculated. After image acquisition, the instrument automatically calculates the measurements of anterior chamber, namely, chamber height endothelium, chamber height epithelium, chamber angle, chamber angle minimum, chamber angle position dependent, chamber angle superior/inferior/nasal/temporal position, and chamber volume $\left(\mathrm{mm}^{3}\right)$. All these measurements were exported into an Excel sheet. The images with poor quality score were excluded.

\section{Measurement of ACV using AS-OCT}

Visante AS-OCT was used to assess the anterior chamber parameters. The instrument scans four meridians and displays it in a single screen, enabling the examiner to visualize eight meridian angles at a time. The scan can be adjusted to visualize other meridians. The default scan meridians are $0^{\circ}-80^{\circ}, 45^{\circ}-225^{\circ}, 90^{\circ}-270^{\circ}$, and $135^{\circ}-315^{\circ}$. All the scans were focused on the pupillary centre to visualize the angles. Twelve scans were taken with $15^{\circ}$ intervals. AS-OCT images image that did not have good quality were excluded as the volume information is dependent on 
image quality. Images with poor reflectivity, poor brightness, poor outline, poor centration, and with no angle insertion were considered of poor quality.

\section{Calculation of ACV}

The images were obtained from the Zeiss Visante AS-OCT. Images were taken directly from the equipment's output function $(816 \times 636$ pixel) in JPEG format.

\section{Image processing}

Images were analysed using MATLAB 7.0 (MathWorks; Natlick, MA, USA) to calculate the ACV. The anterior chamber details alone were cropped, the image was enhanced by noise reduction, and image contrast was improved. The observer marked the pupillary border on either side using the mouse. Once the pupillary border was selected, ACV was calculated based on integration.

The calculation of the entire ACV was similarly done using the shell method of integration. $^{3}$

$$
V=2 \pi \int_{a}^{b} x f(x) d x
$$

Each scan was subjected to this formula and the ACV was calculated. A total of 12 scans per eye was performed and the average of all calculated ACV was calculated. The mean ACV calculated was considered as the final ACV of that eye. All the measurement markings were done by a single trained examiner.

\section{Statistical analysis}

Statistical analysis was performed using SPSS version 14.0 (SPSS Inc.; Chicago, USA) and Medcalc version 8.1.0 (Medcalc software; Mariakerke, Belgium). The Kolmogorov Smirnov test was used to assess the normality of the data. Significance level was kept at 5\%. The independent t-test was done to compare the means of ACV between Pentacam and AS-OCT as well as the mean ACV between

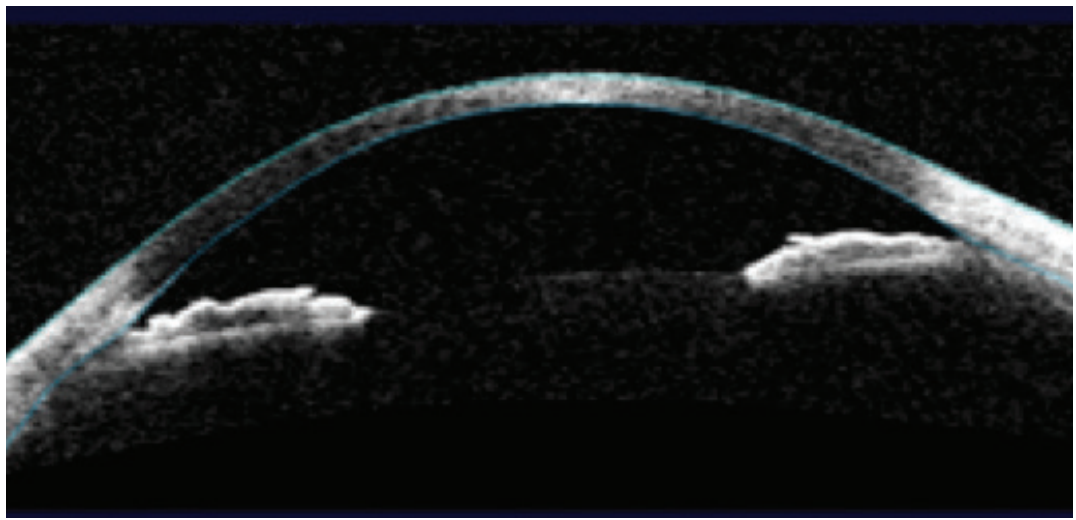

Fig. 1. Input image of the anterior chamber into the Matlab window. 
males and females with these two modalities. Pearson and intra-class correlation (ICC) were performed to find out the correlation of ACV obtained by the two instruments. Bland-Altman analysis was done to find the levels of agreement between the two instruments. Only the right eye was considered for analysis.

\section{Results}

One hundred and nineteen eyes of 119 subjects were considered for analysis. The mean age of the subjects was $42.58 \pm 13.15$ years, out of which 74 were females and 45 were males. The mean ACV volume measured using AS-OCT was $119.17 \pm$ $26.56 \mathrm{~mm}^{3}$ and with Pentacam was $131.29 \pm 34.26 \mathrm{~mm}^{3}$. The mean ACV between males and females is given in Table 1, where no significant difference was found between males and females with these two modalities. The comparison of means of ACV with the two instruments was statistically significant ( $t=-8.857$, Mean Difference $=-12.11,95 \% \mathrm{Cl}:[-14.82,-9.40], \mathrm{p}<0.001)$. The correlation between the chamber volumes between the two instruments was $r=0.91(p<0.0001)$. ICC between the two instruments was $0.94(95 \% \mathrm{Cl}: 0.91,0.96)(p<0.0001)$ with Cronbach's alpha value of $0.94(p<0.0001)$.

Table 1. Comparison of ACV between males and females with the two modalities

\begin{tabular}{|l|l|l|l|l|}
\hline Modalities & $\begin{array}{l}\text { Mean ACV of } \\
\text { males } \\
(\mathbf{M e a n} \pm \mathbf{S D}) \\
\left(\mathbf{m m}^{3}\right)\end{array}$ & $\begin{array}{l}\text { Mean ACV } \\
\text { of females } \\
(\text { Mean } \pm \text { SD) } \\
\left(\mathbf{m m}^{3}\right)\end{array}$ & $\begin{array}{l}\text { Mean Difference } \\
(\mathbf{9 5 \%} \mathbf{C l})^{*}\end{array}$ & p-value \\
\hline Pentacam & $134.08 \pm 36.53$ & $129.59 \pm 32.96$ & $\begin{array}{l}4.494 \\
(-8.364,17.352)\end{array}$ & 0.490 \\
\hline AS-OCT & $121.56 \pm 26.67$ & $117.72 \pm 26.56$ & $\begin{array}{l}3.831 \\
(-6.130,13.792)\end{array}$ & 0.448 \\
\hline
\end{tabular}

ACV: anterior chamber volume; SD: standard deviation; $\mathrm{Cl}$ : confidence intervals; *independent t-test significance level $(p<0.05)$

\section{Limits of agreement between the two instruments}

The Bland-Altman plot showed poor agreement between the chamber volume measurements obtained by Pentacam and AS-OCT with Mean Difference of 12.1 $\mathrm{mm}^{3}$ (shown in Fig. 2) (limits of agreement: 41.4 to -17.1)

\section{Discussion}

ACV measurement provides quantitative information regarding the anterior segment. The current study involves ACV measurement with AS-OCT using Matlab software, which was used to mark the anterior chamber border and then calculate the volume. The current study has a larger sample size in accordance to the ACV in existing literature, as shown in Table 2. 


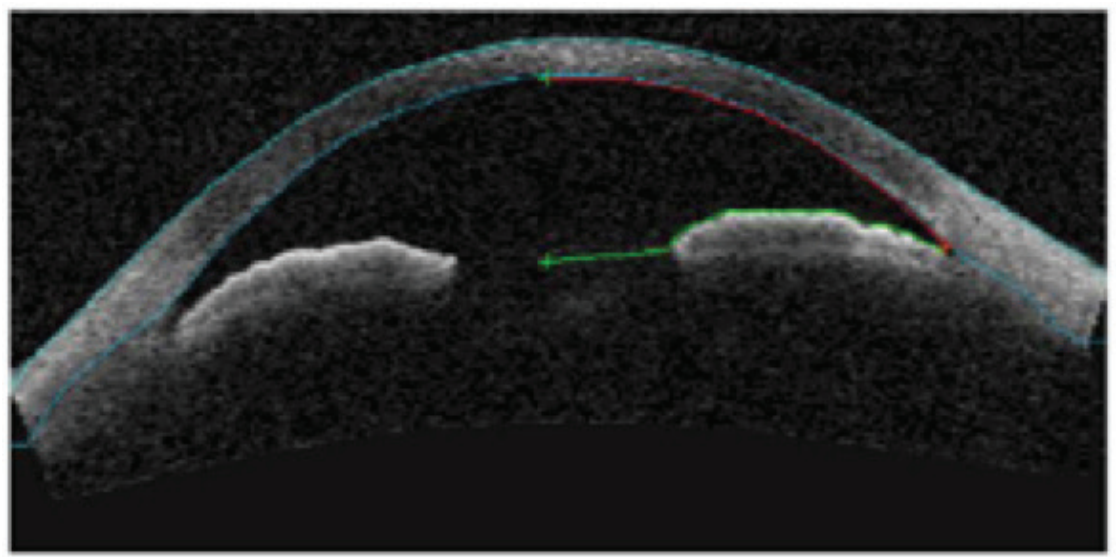

Fig. 2. Marking of anterior the chamber and pupillary border with the calculation of ACV.

Table 2. Comparison of sample size with existing literature

\begin{tabular}{|l|l|}
\hline Study details & Sample size \\
\hline Wang et al. $(2007)^{3}$ & 24 \\
\hline${\text { Labiris et al. }(2009)^{5}}^{5}$ & 20 \\
\hline Fukuda et al. $(2011)^{6}$ & 40 \\
\hline Fu et al. (2010) & 50 \\
\hline Fu et al. (2010) & \\
\hline Current study & 39 \\
\hline
\end{tabular}

Studies have reported the repeatability and reproducibility of these two instruments. $3,5,6$ In our study, the measurements were taken by a single trained examiner. An average of three readings was taken for Pentacam and an average of 12 scans $^{3}$ was considered for AS-OCT. When the averages of these two modalities were compared, the ACV measured by Pentacam was $12.11 \mathrm{~mm}^{3}$ more than that measured by AS-OCT. This finding was consistent with Fukuda et al., ${ }^{6}$ who evaluated the repeatability and reproducibility of ACV obtained using 3-D corneal and anterior segment optical coherence tomography (CAS-OCT) and dual Scheimpflug imaging, where CAS-OCT ACV was $8 \mathrm{~mm}^{3}$ less than Scheimpflug imaging. The smaller difference could be related to CAS-OCT having a mean axial resolution of 11 microns, whereas the Visante AS-OCT has a mean axial resolution of 18 microns. Fu et al. ${ }^{7}$ showed that Pentacam ACV measurements were $14.21 \mu \mathrm{L}$ less than values given by AS-OCT. Labiris et al. ${ }^{5}$ have showed that there is no difference between ACV measured by Pentacam and AS-OCT. Our study found a difference in ACV as measured between the two modalities. Furthermore, the 


\section{Correlation Between Pentacam and ASOCT}

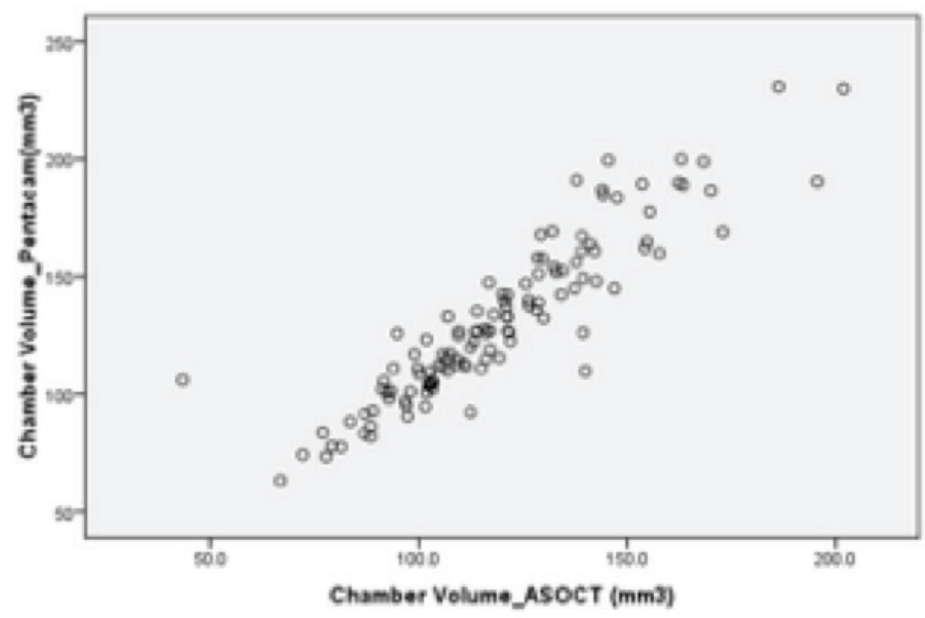

Fig. 3. Scatter plot showing the correlation of ACV measurements between Pentacam and AS-OCT.

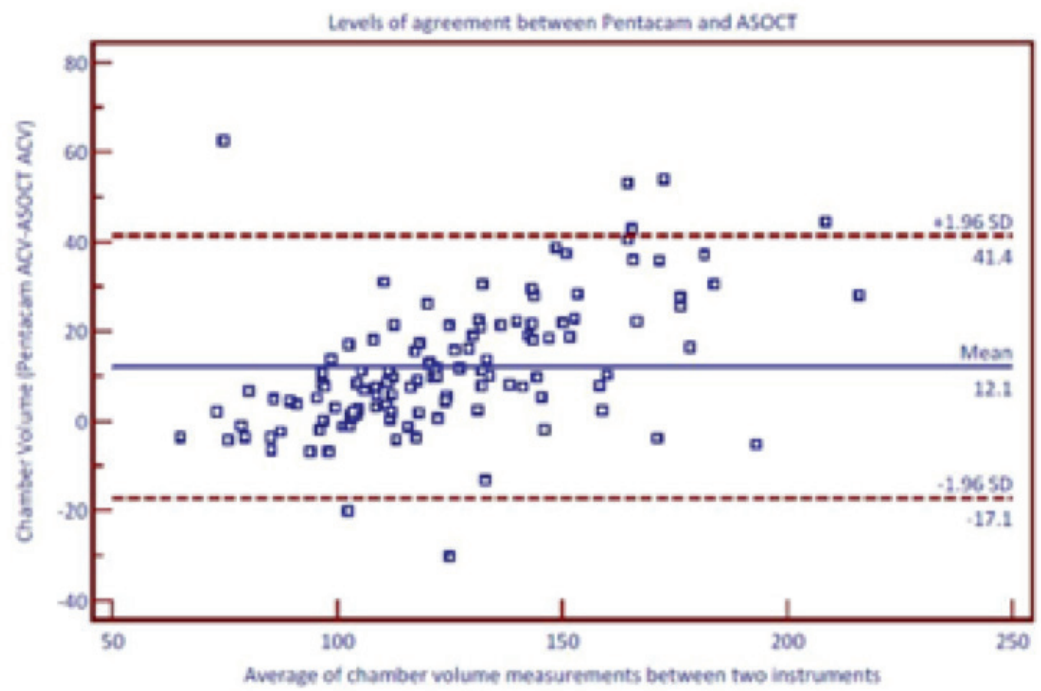

Fig. 4. Bland-Altman plot of difference in chamber volume measurements between Pentacam and AS-OCT and average of chamber volume measurements between the two measurements. 
mean ACV values found in our study were less when compared to other studies. This could be due to the shorter axial lengths and shallower anterior chamber depths in Indian eyes compared to other ethnicities..$^{8,9}$ In our study, no significant difference in ACV was noted between males and females; however, true values of ACV for females were lesser than males. Another study by Fu et al. ${ }^{10}$ also proposed that Pentacam ACV measurements are less than AS-OCT ACV measurements by $11.8 \mu \mathrm{L}$. The differences in mean difference between these studies are seen because of the varied sample size of the two studies. The difference between Pentacam and AS-OCT measurements found in our current study could possibly be due to the ethnic differences between the Chinese and Indian population. ${ }^{9}$

There was a poor agreement between the ACV measured by Pentacam and AS-OCT. The mean difference was $12.11 \mathrm{~mm}^{3}$, with wider limits of agreements from $-17.1 \mathrm{~mm}^{3}$ to $41.4 \mathrm{~mm}^{3}$, which fall outside clinically acceptable levels. ${ }^{6,7,10}$ The measurements of ACV with Pentacam and AS-OCT correlated well ( $r=0.91$, $p<0.001$ ) and were repeatable, with an ICC value of 0.94 . Although the ACV measured by these instruments is repeatable, it is not interchangeable due to the large difference in mean values and the large width in confidence interval limits. This could be due to the differences in measurement methods between the two devices. Pentacam uses the Scheimpflug principle, where the light of the instrument does not penetrate the corneoscleral limbus, thereby giving interpolated values by extending the lines of the cornea and iris border with that of the angle, which can overestimate ACV measurements. In our study, both the comparison of means and levels of agreement analysis showed that the measurements of ACV given by these modalities were statistically different, in accordance with studies by Fu et al.,10 Apart from all, this study provides a better estimate of ACV when compared to other studies. Our study has a few limitations. Intersession repeatability between the two instruments was not studied, which could explain the difference in ACV over a period of time (diurnal variation) examined by a single examiner. Although the sample size was large when compared to other studies, there is no normative data reference for the mean values of different ethnicities and thus, grading of shallow or deep eyes becomes difficult. The ACV calculated could be erroneous if the image was decentred or of poor quality. If image scaling is irregular, the calculation of the volume would be highly variable.

\section{Conclusion}

AS-OCT can be used to measure ACV since the measurements provided by these modalities correlate with each other. However, the levels of agreement between these modalities are not sufficient to allow these instruments to be comparable. 


\section{References}

1. Johnson SB, Passmore JA, Brubaker RF. The fluorescein distribution volume of the anterior chamber. Invest Ophthalmol Vis Sci. 1977;16(7):633.

2. Rabsilber TM, Khoramnia R, Auffarth GU. Anterior chamber measurements using Pentacam rotating Scheimpflug camera. J Cataract Refract Surg. 2006;32(3):456-459.

3. Wang N, Wang B, Zhai G, Lei K, Wang L, Congdon N. A method of measuring anterior chamber volume using the anterior segment optical coherence tomographer and specialized software. Am J Ophthalmol. 2007;143(5):879-881.

4. Yi JH, Lee $\mathrm{H}$, Hong S, et al. Anterior chamber measurements by Pentacam and AS-OCT in eyes with normal open angles. Korean J Ophthalmol. 2008;22(4):242-245.

5. Labiris G, Gkika M, Katsanos A, Fanariotis M, Alvanos E, Kozobolis V. Anterior chamber volume measurements with Visante optical coherence tomography and Pentacam: repeatability and level of agreement. Clin Exp Ophthalmol. 2009;37(8):772-774.

6. Fukuda S, Kawana K, Yasuno Y, Oshika T. Repeatability and reproducibility of anterior chamber volume measurements using 3-dimensional corneal and anterior segment optical coherence tomography. J Cataract Refract Surg. 2011;37(3):461-468.

7. Fu J, Wang X, Li S, Wu G, Wang N. Comparative study of anterior segment measurement with Pentacam and anterior segment optical coherence tomography. Can J Ophthalmol. 2010;45(6):627-631.

8. Jonas JB, Nangia V, Gupta R, et al. Anterior chamber depth and its associations with ocular and general parameters in adults. Clin Exp Ophthalmol. 2012;40(6):550-556.

9. Yin $\mathrm{G}$, Wang $\mathrm{YX}$, Zheng $\mathrm{ZY}$, Yang $\mathrm{H}, \mathrm{Xu} \mathrm{L}$, Jonas, JB. Ocular axial length and its associations in Chinese: the Beijing Eye Study. PloS One. 2012. doi:10.1371/journal.pone.0043172.

10. Fu J, Li SN, Wang XZ, et al. Measurement of anterior chamber volume with rotating Scheimpflug camera and anterior segment optical coherence tomography. Chin Med J. 2010;123(2):203-207. 